\title{
Infection Prevention Strategies in Cardiac Rehabilitation [1]-A Behavioral Intervention for Patients [2]
}

\author{
Wendy Bjerke \\ Sacred Heart University, Fairfield, USA \\ Email:bjerkew@sacredheart.edu
}

How to cite this paper: Bjerke, W. (2017) Infection Prevention Strategies in Cardiac Rehabilitation [1]-A Behavioral Intervention for Patients [2]. Health, 9, 1273-1284. https://doi.org/10.4236/health.2017.99092

Received: June 21, 2017

Accepted: September 3, 2017

Published: September 6, 2017

Copyright $\odot 2017$ by author and Scientific Research Publishing Inc. This work is licensed under the Creative Commons Attribution International License (CC BY 4.0).

http://creativecommons.org/licenses/by/4.0/

\begin{abstract}
Background: Healthcare Acquired Infections (HAI) result in over 100 thousand deaths each year with one third of these deaths preventable via behaviors such as hand washing among health care providers in inpatient settings. Less research has been conducted in outpatient exercise settings such as cardiac rehabilitation (CR) among patients. Purpose: The purpose of this study was to examine the effectiveness of HAI prevention strategies in a CR setting among patients. Methods: Observations of the frequency of hand washing among CR patients pre and post four HAI strategies including provision of HAI education and signs, hand washing demonstrations, a HAI prevention video, and hand sanitizer samples. Washing hands prior to CR (WI) was observed as well as washing hands prior to leaving the $\mathrm{CR}$ center (WO). Methods included recording the frequency of WI and WO among all patients at baseline and after each of the four interventions. Mean frequencies of WI and WO were compared among a mean of 22 - 43 CR patient visits over 12 weeks using descriptive statistics and t-tests to determine if changes were significant pre and post intervention strategies. Results: At baseline, no patients WI or WO during an outpatient CR visit. Post interventions $1-4$, the percentage of patients WI and WO was 33 and 34, 32 and 26, 32 and 29, 33 and 22 respectively. At a one-year follow up, the percentage of patients WI and WO was $40 \%$. Conclusion: Increases in frequency and the percentage of WI and WO were observed among patients meriting continued examination of HAI prevention strategies among patients in outpatient exercise settings such as CR.
\end{abstract}

\section{Keywords}

Healthcare Acquired Infections, Out-Patient Settings, Prevention, Behavior Modification 


\section{Introduction and Background}

Annually in the United States, it is estimated that 1.7 million patients acquire a health care associated infection (HAI), defined as an infection acquired in a hospital or health care facility which results in approximately one hundred thousand deaths [1]. Of 700 thousand HAI's that took place in hospital settings in 2011, 75 thousand deaths were the result [2]. In addition, one-third to three-fourths of these infections may be preventable [3]. Approximately $90 \%$ of HAI's are of bacterial origin with routes of transmission including contact, droplet, airborne, or through a common vehicle such as food or medical equipment [4]. Examples of common HAI's include staph infections and illnesses such as pneumonia. Common sources include medical equipment such as catheters [5]. Most researchers including the Centers for Disease Control and Prevention [2] have compiled data primarily associated with inpatient settings despite increasing trends in treatment of patients in outpatient centers and no formal studies have assessed patient behaviors specifically within the context of preventing HAI's though recommendations have been suggested [6].

Schimier [7] is among researchers who argue that approximately three-fourths of HAI's are preventable. An aggregate analysis of 30 multi-modal studies found that HAI rates could be reduced by $10 \%-70 \%$ with prevention and control strategies [1]. Strategies such as frequent hand washing and provision of sanitizing equipment are well known among most health care professionals with specific recommendations for outpatient settings proposed by the CDC [8] but awareness of systematic approaches pursuant to reducing infection rates among health care providers and patients are less prevalent, especially in outpatient settings including Cardiac Rehabilitation (CR). In fact, a recent examination of HAI prevention strategies in outpatient settings did not include any $\mathrm{CR}$ or rehabilitation centers or facilities similar to CR in the assessment [6].

A systematic approach includes but is not limited to optimal surveillance of HAI's, use of trained infection control public health and/or allied health professionals, feedback mechanisms, and use of a dedicated epidemiologist. In addition, knowledge of risk factors, environmental variables, and identification of increased risk groups as well as the most common sources and types of HAI's are required to reduce infection rates [4]. Considerations related to the present study include the applicability of these strategies to patient populations in outpatient settings such as CR.

Identified risk factors for HAI's that are relevant to CR patients include the type or severity of diagnosis at admission and specific risk factors include advanced age, comorbid factors, and nutrition status. Chief among risk factors is advanced age with between $40 \%-60 \%$ of all HAI's occurring in patients over 65 years of age [9]. Additional variables found to increase risk particularly among CR patients include infection risk post bypass surgery or angioplasty procedures if healing from those procedures is delayed [1]. Though the most common types of infections include urinary tract infections, pneumonia, infections at the surgical site, bloodstream infections, and central nervous system infection [2] res- 
piratory illnesses such as the flu or upper respiratory tract infections are particularly relevant to CR settings especially within the context of adherence rates for influenza and pneumonia vaccinations which varied in 2015 between 35 and $65 \%$ among the age ranges typically observed in CR [10].

Hand hygiene practices and in particular hand washing among health care professionals was identified as a compelling factor in the promotion of health and reduction of infections as early as the 1840's by Dr. Semmelweis in his comparison of health care setting and home setting differences in maternal fatality rates after childbirth [11]. Hand hygiene practices including hand washing and sanitizing with an alcohol-based cleanser are widely recognized methods of preventing and reducing the prevalence of HAI's in health care settings [12] despite HAI's remaining a top cause of death in the United States. Hand hygiene has been at the forefront of infection control strategies due to its simplicity in nature and influential outcomes however, the compliance rate in CR outpatient settings for both staff and patients has not been documented outside of reports at professional conferences [13]. Umscheidand colleagues [1] observed after an extensive review of infection prevention strategies that up to $75 \%$ of HAI's, including pneumonia, flu, and upper respiratory tract infections, could be prevented via hand washing and sanitizing equipment.

The majority of HAI related research has been conducted in inpatient settings. As a result, outpatient settings including ambulatory care centers and rehabilitation settings are underrepresented relative to assessment and research. In fact, researchers conducting one of the few studies examining infection control effectiveness in outpatient ambulatory centers found that $67 \%$ of the 68 ambulatory care centers had at least one lapse in infection control during the year of assessment [12]. Additionally, no publications were found that assess infection related interventions and behaviors among patients in CR settings though research has been disseminated in other settings such as poster presentations at professional peer reviewed conferences [13]. Outpatient or ambulatory centers were included in Son and colleague's [14] three-year intervention and assessment of 19 inpatient hospital departments and 15 outpatient centers with marked overall improvement in compliance among staff from $60 \%$ up to $97 \%$.

CR characterizes outpatient programs that treat patients after a variety of acute and chronic conditions including myocardial infarction, coronary bypass graft procedure, angioplasty, and congestive heart failure [15]. CR includes supervised exercise and also nutrition education, stress management, and other services that address holistic health variables [16]. Though only between $14 \%$ $35 \%$ of eligible patients are referred to CR programs, this patient population ranges between 100,000 and 275,000 in number [17]. Though the average age of $\mathrm{CR}$ patients is above 60, age ranges vary between children and adults older than 100 years of age [18]. Recently published consensus statements continue to promote the cost effectiveness of CR programs relative to other tests and procedures [16]. Given the prevalence of HAI's, the increased number of HAI risk factors among CR patients, and the outpatient often hospital based setting of 
many CR programs, it is relevant to examine this topic and the outcomes of infection prevention strategies in CR. Implications of these findings could be applied to numerous outpatient settings including respiratory therapy, physical therapy, and occupational therapy among others.

CR programs are relevant venues for infection prevention strategies given the relative immunodeficiency among patients with chronic disease [3], and increased infection risk associated with invasive cardiac procedures [19]. Any hospital based outpatient setting potentially exposes patients to hospital based as well as community based infections. Additionally, all previously described studies assess staff hand hygiene behaviors, and this study assessed behaviors among patients.

\section{Setting and Participants}

A hospital in Connecticut USA promoting HAI prevention strategies and policies in outpatient settings among hospital staff was the site for the study given that prevention strategies in place for staff were applied to and assessed among patients for the first time. A total of 69 patients were observed during the study; Forty-three patients during an initial data collection period in 2012 and 26 during a second observation in 2013. Sixty-two percent of the patients were male with an average age of 61 . The remaining patients were female with an average age of 64. IRB approval for retrospective analysis of aggregate de-identified data was obtained at the target hospital as well as from the collaborating university. All attending patients during the data collection period were observed; however, attendance varied for patients. The data collection period was directed by the duration of a clinical placement of a student in allied health and associated clinical instructor who were the individuals collecting observations for the department.

\section{Interventions and Methods}

The CR staff and interns were charged with providing education, interventions, and were routinely collecting data relative to HAI prevention behaviors among staff and patients for the purpose of outcomes analysis. Specifically, the CR staff consisted of nurses, exercise physiologists, nutritionists, and student interns who were previously trained in HAI prevention strategies by the hospital. These identical strategies were incorporated into existing CR educational programming for patient education. The educational programming occurred in between exercise sessions and included a video detailing hygiene behavior related to HAI's, and orientation to signs and hand washing stations added to the CR gym/room specifically for patient use. Baseline data was collected for 4 weeks prior to all interventions. Intervention 1 took place during week 5 and 6 . Intervention 2 took place during week 7 and 8 . Intervention 3 took place during week 9 and 10 and intervention 4 took place during week 11 and 12 of the 2012 data collection period. Data was collected 12 weeks from May to August 2012 with a 
follow up data collection period one year later in August 2013 for two weeks to determine the effectiveness of four infection prevention strategies on washing in (WI) and/or washing out (WO) behaviors among patients.

The specific interventions provided during CR sessions included:

1) Signs to promote hand washing and accessible cleaning stations.

2) "Bioderm" demonstration of hand washing techniques.

3) Educational video.

4) Provision of sanitizer samples.

Table 1 provides additional details including the timing of all interventions. All interventions were provided during the 2012 data collection period. Interventions 1 and 3 were provided during the 2013 data collection period. Figure 1 displays the provision of hand sanitizer in the CR center.

Quantitative data was analyzed pre and during the initial 12-week intervention period and included the mean number and \% of patients WI and WO of the CR room. Follow up data was collected one year later to assess longer term effectiveness of the program. At that time intervention strategies 1 and 3 were assessed among a new patient cohort. Specifically, the percentage of patients observed WI or WO for six days within two weeks was reported in August 2013 and compared to data collected in August 2012. Descriptive statistical analysis was applied to assess the percent of patients adopting hand washing behaviors pre and post interventions and for the one year follow up period. Descriptive and inferential statistical analysis using PASW Statistics 18 was used to assess the observed changes in hand washing behaviors among patients. Specifically, unpaired $t$ tests were used to assess mean changes during the initial 12-week intervention period.

Table 1. Summary of NI prevention patient interventions.

\begin{tabular}{|c|c|c|}
\hline Intervention & Administration & Timing of Intervention \\
\hline $\begin{array}{l}\text { 1. Signs }+ \text { Cleaning Stations } \\
\text { (Figure 1) }\end{array}$ & $\begin{array}{c}\text { Signs reminding patients } \\
\text { to wash equipment and } \\
\text { hands posted in CR room/gym. } \\
\text { Sinks made available } \\
\text { and inviting to patients. } \\
\text { Sanitizer bottles provided }\end{array}$ & $\begin{array}{l}\text { Provided during week } 5 \text { - } 6 \\
\text { and one year follow-up. }\end{array}$ \\
\hline 2. Bioderm Demonstration & $\begin{array}{l}\text { CR staff and patient } \\
\text { volunteers participate in } \\
\text { Bioderm demonstration } \\
\text { emphaizing hand washing } \\
\text { techniques and effectiveness. }\end{array}$ & Provided during week 7 - 8. \\
\hline 3. Educational Video & $\begin{array}{c}\text { “All Washed Up” (Vital Smarts) } \\
\text { video shown (6 minutes } \\
\text { duration) during week } 2 .\end{array}$ & $\begin{array}{l}\text { Provided during week } 9 \text { - } 10 \\
\text { and one year follow-up. }\end{array}$ \\
\hline $\begin{array}{l}\text { 4. Provision of Sanitizer } \\
\text { Samples }\end{array}$ & $\begin{array}{c}\text { Samples of sanitizers were given } \\
\text { during week }\end{array}$ & $\begin{array}{l}\text { Provided during } \\
\text { week } 11-12 .\end{array}$ \\
\hline
\end{tabular}




\section{Findings}

Prior to hand washing behavior interventions, no CR patients were observed WI or WO. For all interventions associated with the initial 12-week data collection period, the percent of patients WI and WO increased ranging between $22 \%$ and $34 \%$. For interventions 1 - 4 respectively, the average number and ranges of patient visits was 32 (22 - 39), 39 (34 - 43), 35 (32 - 36), and 37 (31 - 41). The percentages of patients WI and WO for interventions 1 - 4 respectively were 33 and 34, 32 and 26, 32 and 29, and 33 and 22. The average percentage for all four interventions was 33 (WI) and 28 (WO). In summary, almost one third of patients were washing in and washing out post any and all interventions compared to no patients demonstrating these behaviors at baseline. One year later in the month of August, patients were observed once again. At that time interventions 1 and 3 were provided or in place. At that time between $39 \%$ and $41 \%$ of patients were observed WI and WO. Specifically, post intervention one, $41 \%$ of patients was observed both WI and WO. Post intervention 3, 39\% of patients were observed WI and WO. In short, this increase from zero to over one third of the CR patients WI or WO appeared to be consistently maintained. Please refer to Table 2 and 3. Significant changes in hand washing behavior were observed when all strategies were assessed in aggregate $(p=0.03)$ and separately significant changes were observed with signs + sanitizer placement, video presentation, and Bioderm demonstration of hand washing techniques. Table 3 depicts the percentage of total patients WI and WO with percentages ranging from $20 \%-38 \%$ post the intervention strategies. One year later, a higher percentage of patients $(40 \%)$ were observed WI or WO of the rehabilitation center (Table 4).

\section{Discussion}

Changes in hand washing behaviors among patients were observed both short term (after 12 weeks of the initial study) and long term (after a year of chronic exposure to select interventions). Generally, the changes we observed were expected. For example, at baseline though some infection prevention strategies applied to staff could have had a potential impact on patient behaviors, this did not appear to be the case given that no patients were observed washing their hands pre or post CR for four weeks. Once the interventions began, this percentage increased from zero to $33 \%$ and $34 \%$. Reported previously was a $39 \%-41 \%$ WI

Table 2. Mean frequency of cardiac rehabilitation patients "Washing in and out".

\begin{tabular}{cccc}
\hline & Washing In (WI) & Washing Out (WO) & P value (WI/WO) \\
\hline Baseline & 0 & 0 & NA \\
Signs + Sanitizer & 10 & 10 & $0.037 / \mathrm{NS}$ \\
"Bioderm" Demo & 12 & 10 & $\mathrm{NS}$ \\
Video & 11 & 10 & $0.01 / 0.05$ \\
Sanitizer Samples & 9 & 8 & $0.00 / 0.03$
\end{tabular}


Table 3. Percentage of cardiac rehabilitation patients "Washing in and out".

\begin{tabular}{|c|c|c|c|}
\hline & $\begin{array}{l}\text { Number of } \\
\text { Patients }\end{array}$ & \% Washing In (WI) & $\%$ Washing Out (WO) \\
\hline \multirow{2}{*}{ Baseline } & Mean $=36$ & & \\
\hline & Range $=22-43$ & 0 & 0 \\
\hline \multirow{2}{*}{ Signs + Sanitizer } & Mean $=32$ & & \\
\hline & Range $=22-39$ & 33 & 34 \\
\hline \multirow{2}{*}{ "Bioderm" Demo } & Mean $=39$ & & \\
\hline & Range $=34-43$ & 32 & 26 \\
\hline \multirow{2}{*}{ Video } & Mean $=35$ & & \\
\hline & Range $=32-36$ & 32 & 29 \\
\hline \multirow{2}{*}{ Sanitizer Samples } & Mean $=37$ & & \\
\hline & Range $=31-41$ & 33 & 22 \\
\hline
\end{tabular}

Table 4. Hand washing frequency (WI or WO) 1 year follow-up.

\begin{tabular}{cccc}
\hline & Pre & Post & P value \\
\hline Mean & 9 & 7 & NS \\
Percentage & 40 & 40 & NA \\
\hline
\end{tabular}

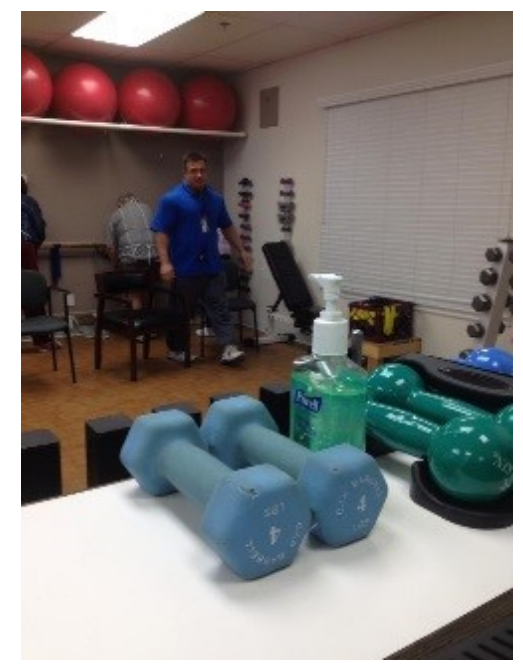

Figure 1. Provision of sanitizer in CR setting.

and WO among patients one year after the initial interventions.

These percentages share some commonalities with other publications examining this topic in health care settings among staff. For example, Erasmus and colleagues [20] conducted a systematic review of hand washing compliance studies in health care settings published through 2008 . Though over $60 \%$ of the 96 studies took place in intensive care settings, the other studies took place in a variety of in-patient and out-patient health care settings. Thirty to 40 percent of staff washed hands pre or post procedures or between patients in intensive care units compared to $50 \%-60 \%$ outside of intensive care units. Additionally, com- 
pliance was greater among nurses (48\%) compared to doctors (32\%).

More recently, Thompson and colleagues [6] assessed overall HAI infection strategies specifically in outpatient settings. Though rehabilitation and exercise settings were not included among the 15 different types of outpatient settings, this study did share in common the incorporation of allied health students in the observations and data collection. Second year medical students were charged with becoming familiar with recent CDC guidelines specific to outpatient settings and assessing compliance among health care professionals. Among the strategies assessed was a hand hygiene guideline including provision of hand washing supplies, hand-washing behavior before and after patient contact, and after contact with body fluids, supplies, and equipment. Though patient behaviors were not assessed, it is relevant to the present study to note that among the medical student recommendations for all outpatient settings was to direct hand hygiene practices and promotion to patients as well as staff. Results among staff were compelling with approximately $90 \%$ compliance to CDC recommended hand hygiene guidelines. Given the comparatively modest compliance observed in the present study, continued interventions are needed if patient behaviors are to be modified more consistently.

Given that the impetus for this study is underrepresentation of outpatient settings relative to handwashing compliance and behaviors as well as an emphasis on patient behaviors, a recent examination of the role of patients in HAI's is also relevant to this study. For example, 700 patients were questioned about the patient's current and potential role in impacting HAI's. Fifty-seven percent of individuals surveyed were not likely to question medical staff about whether or not they washed their hands prior to a treatment of office visit, $43 \%$ of in-patients responded similarly and reported that they "trusted" that hospital staff was compliant with HAI prevention strategies. Twenty percent of individuals surveyed reported that they would not pose any question out of fear that they would offend the health care provider, yet over $70 \%$ of all subjects reported that patients could potentially improve HAI infection rates if they posed these types of questions in advance of treatments and procedures [21]. Findings from this study suggest that patients have the potential to progress beyond questioning health care providers about HAI prevention strategies by implementing hand-washing behaviors themselves.

An additional consideration is the relationship between this study and the World Health Organization's (WHO) multimodal strategies associated with hand hygiene. Among strategies includes "patient empowerment" interventions, programs, and initiatives including previously described involvement among patients in the direct questioning of health care providers relative to hygiene compliance but also inclusive of more active roles such as mentoring [22]. The activities and outcomes associated with this study potentially empower patients to have a direct impact on HAI prevention strategies and NI rates in health care facilities. 
A noteworthy anecdotal observation during data collection of a patient refusing to comply with recommendations and interventions calls attention to the need to gain an understanding of the complex issues associated with infectious disease control. Specifically, this patient argued that use of sanitizers and frequent hand washing in the CR setting was potentially contributing to the increase in resistance among bacteria to anti-bacterial agents. This is a valid point but made in the wrong setting and context. Though researchers are examining use or overuse of antibacterial agents of all types as negatively impacting health, the environment where infectious agents require control should be carefully considered. For example, in environments where the majority of individuals are in good health; for example, a fitness center, it may actually benefit the community's health to clean and sanitize areas with less vigilance. However, in areas where a high proportion of individuals may be ill, it remains best practice to comply with all infectious agent prevention strategies and recommendations including hand washing and sanitizer use in outpatient settings [23].

Limitations of the study include the retrospective analysis of data that had already been collected by CR staff and student interns. Inconsistent numbers of data collection days and varied numbers of patients during observation days can potentially reduce the implications of findings and argument that these results would be repeatable in other CR settings. However, despite these inconstancies, it is relevant to emphasize that the \% of patients adopting and adhering to HAI prevention strategies was generally consistent at approximately one third. This was also observed during the follow up data collection and supported by an analysis of variance among proportions of patients WI and WO.

This study achieves the objective of providing an example of outpatient infection prevention strategies including assessment of the outcome behaviors among patients. Anecdotal observations among staff were made pursuant to future studies and improvement of patient outcomes. These included timing the interventions to coincide with cold and influenza season as well as modifying signs periodically to attract the attention of patients. Additionally, the provision of the video and Bioderm intervention could be done online. Follow up studies comparing the effectiveness of strategies among patients to staff could be appropriate directions for future research as well as more examinations of HAI prevention strategies in novel settings and among populations outside of health care providers.

This study contributes to an argued gap in the literature relative to assessment of HAI prevention strategies in outpatient settings [6]. In addition to the novel setting for this study (CR), the assessment of patient behaviors is also unique relative to current literature. Given that hand washing behaviors did change among patients, implications of these findings include the possibility that infection prevention strategies directed towards health care professionals could also be applied to patients. For example, Maskerine and colleagues [24] identified several inhibiting and promoting factors for hand washing adherence among health care professionals including access to hand washing stations, provision of 
time for hand washing, and specified benefits. These factors have been associated with specific behavior change theories in health care workers and could also apply to patients [25]. Among health care professionals, the health belief model, theory of reasoned action, and planned behavior are all associated with adherence to hand washing behaviors [25]. These theories associate increased hand hygiene with a belief that the behavior will result in a positive outcome. Similarly, this association could motivate patients to comply with HAI infection prevention strategies in outpatient settings. Given the continued mortality rate associated with HAI's [25] and an estimated health care cost savings of between 140 million and 4.25 billion dollars per year associated with HAI prevention strategies (Schimier, 2016), directing interventions to patients as well as health care providers can potentially and further prevent HAI'S and promote health in outpatient settings [26].

\section{Conclusion}

Prior to the assessed hand washing behavior interventions, no CR patients were observed washing their hands prior to or after completion of their CR session compared to over one third of patients WI or WO after a series of provided and varied interventions including signs, motivating factors, and educational videos. Feedback mechanisms resulting from the findings of surveillance are critical to prevention and control and may be one of the least applied yet most effective strategies [27]. Outcomes from studies of infection control strategies such as this one could direct the role of a hospital based epidemiologist potentially coordinating all of the previously described strategies of surveillance, identification of risk factors and prevalent infections, protection of health care workers and patients, and rapid dissemination of information resulting from surveillance. Given that the provision of feedback is suggested, the examination of HAI infection strategies outcomes in this study could benefit the patients and staff of CR centers and be applied to other rehabilitation settings such as physical therapy, occupational therapy, and therapeutic fitness centers.

\section{References}

[1] Umscheid, C.A., Rajender, A., Williams, K. and Brennan, P. (2011) Estimating the Proportion of Healthcare-Associated Infections That Are Reasonably Preventable and the Related Mortality and Costs. Infection Control and Hospital Epidemiology, 32, 101-114. https://doi.org/10.1086/657912

[2] Centers for Disease Control and Prevention (2016) HAI Surveillance. http://www.cdc.gov/hai/surveillance/

[3] Magill, S.S., Edwards, J.R., Bamberg, W., Beldavs, Z.G., Dumyati, G., Kainer, M. A., Ray, S.M., et al. (2014) Multistate Point-Prevalence Survey of Health Care-Associated Infections. New England Journal of Medicine, 370, 1198-1208. https://doi.org/10.1056/NEJMoa1306801

[4] Nelson, K. and Williams, C. (2007) Infectious Disease Epidemiology: Theory and Practice. Jones and Bartlett, Sudbury.

[5] Lobdell, K.W., Stamou, S. and Sanchez, J.A. (2012) Hospital-Acquired Infections. 
Surgical Clinics of North America, 92, 65-77. https://doi.org/10.1016/j.suc.2011.11.003

[6] Thompson, D., Bowdey, L., Brett, M. and Cheek, J. (2016) Using Medical Student Observers of Infection Prevention, Hand Hygiene, and Injection Safety in Outpatient Settings: A Cross-Sectional Survey. American Journal of Infection Control, 44, 374-380. https://doi.org/10.1016/j.ajic.2015.11.029

[7] Schmier, J.K., Hulme-Lowe, C.K., Semenova, S., Klenk, J.A., DeLeo, P.C., Sedlak, R. and Carlson, P.A. (2016) Estimated Hospital Costs Associated with Preventable Health Care-Associated Infections If Health Care Antiseptic Products Were Unavailable. ClinicoEconomics and Outcomes Research: CEOR, 8, 197. https://doi.org/10.2147/CEOR.S102505

[8] Centers for Disease Control and Prevention (2016) Outpatient Care HAI Guidelines. https://www.cdc.gov/HAI/settings/outpatient/outpatient-care-guidelines.html

[9] Avci, M., Ozgenc, O., Coskuner, S.A. and Olut, A.I. (2012) Hospital Acquired Infections (HAI) in the Elderly: Comparison with the Younger Patients. Archives of Gerontology and Geriatrics, 54, 247-250.

https://doi.org/10.1016/j.archger.2011.03.014

[10] Centers for Disease Control and Prevention (2016) Influenza. http://www.cdc.gov/flu/protect/vaccine/index.htm.

[11] Biddle, C. (2009) Semmelweis Revisited: Hand Hygiene and Nosocomial Disease Transmission in the Anesthesia Workstation. AANA Journal, 77, No. 3.

[12] Schaefer, M., Jhung, M., Dahl, M., Schille, S., Simpson, C., Llata, E., et al. (2010) Infection Control Assessment of Ambulatory Surgical Centers. JAMA, 303, 2273-2279. https://doi.org/10.1001/jama.2010.744

[13] Bjerke, W., Standish, S. and Pastore, A. (2013) Infection Prevention Strategies in Cardiac Rehabilitation Programs. Poster Presented at the American Association of Cardiovascular and Pulmonary Rehabilitation October 3-5, Nashville.

[14] Son, C., Chuck, T., Childers, T., Usiak, S., Dowling, M., Andiel, C., et al. (2011) Practically Speaking: Rethinking Hand Hygiene Improvement Programs in Health Care Settings. American Journal of Infection Control, 39, 716-724. https://doi.org/10.1016/j.ajic.2010.12.008

[15] Grace, S.L., Turk-Adawi, K.I., Contractor, A., Atrey, A., Campbell, N.R., Derman, W., Buckley, J., et al. (2016) Cardiac Rehabilitation Delivery Model for Low-Resource Settings: An International Council of Cardiovascular Prevention and Rehabilitation Consensus Statement. Progress in Cardiovascular Diseases, 59, 303-322. https://doi.org/10.1016/j.pcad.2016.08.004

[16] Lavie, C.J., Arena, R. and Franklin, B.A. (2016) Cardiac Rehabilitation and Healthy Life-Style Interventions: Rectifying Program Deficiencies to Improve Patient Outcomes. American Journal of Infection Control, 67, 13-15. https://doi.org/10.1016/j.jacc.2015.09.103

[17] Balady, G., Ades, P., Bittner, V., Franklin, B., Gordon, N., Thomas, R., et al. (2011) Referral, Enrollment, and Delivery of Cardiac Rehabilitation/Secondary Prevention Programs at Clinical Centers and beyond a Presidential Advisory from the American Heart Association. Circulation, 124, 2951-2960. https://doi.org/10.1161/CIR.0b013e31823b21e2

[18] Beatty, A.L., Li, S., Thomas, L., Amsterdam, E.A., Alexander, K.P. and Whooley, M.A. (2014) Trends in Referral to Cardiac Rehabilitation after Myocardial Infarction: Data from the National Cardiovascular Data Registry 2007 to 2012. Journal of the American College of Cardiology, 63, 2582-2583. 
https://doi.org/10.1016/j.jacc.2014.03.030

[19] Lutarewych, M., Morgan, P. and Hall, M. (2004) Improving Outcomes of Coronary Artery Bypass Graft Infections with Multiple Interventions: Putting Science and Data to the Test. Infection Control \& Hospital Epidemiology, 25, 517-519. https://doi.org/10.1086/502433

[20] Erasmus, V., Daha, T., Brug, H., Hendrik, J., Richardus, M., Behrendt, M., et al. (2010) Systematic Review of Studies on Compliance with Hand Hygiene Guidelines in Hospital Care. Infection Control, 31, 283-294. https://doi.org/10.1086/650451

[21] Pittet, D., Panesar, S., Wilson, K., Yongtin, Y., Morris, T., et al. (2011) Involving the Patient to Ask about Hospital Hand Hygiene: A National Patient Safety Agency feasibility study. Journal of Hospital Infection, 77, 299-303.

https://doi.org/10.1016/j.jhin.2010.10.013

[22] McGuckin, M., Storr, J., Longtin, Y., Allegranzi, B., Pittet, D., et al. (2011) Patient Empowerment and Multimodal Hand Hygiene Promotion: A Win-Win Strategy. American Journal of Medical Quality, 26, 10-17. https://doi.org/10.1177/1062860610373138

[23] Blaser, M. (2014) Missing Microbes: How the Overuse of Antibiotics Is Fueling Our Modern Plagues. Macmillan, New York.

[24] Maskerine, C. and Loeb, M. (2006) Improving Adherence to Hand Hygiene among Health Care Workers. The Journal of Continuing Education in the Health Professions, 26, 244-251. https://doi.org/10.1002/chp.77

[25] Michie, S. and Johnson, M. (2010) Theories and Techniques of Behavior Change: Developing a Cumulative Science of Behavior Change. Health Psychology Review, 6, 1-6. https://doi.org/10.1080/17437199.2012.654964

[26] McGuckin, M., Govednik, J., Hyman, D. and Black, B. (2013) Public Reporting of Health Care-Associated Infection Rates Are Consumers Aware and Engaged?. American Journal of Medical Quality, 29, No. 1.

[27] Harbarth, S., Sax, H. and Gastmeier, P. (2003) The Preventable Proportion of Nosocomial Infections. Journal of Hospital Infection, 54, 258-260.

https://doi.org/10.1016/S0195-6701(03)00150-6 
Submit or recommend next manuscript to SCIRP and we will provide best service for you:

Accepting pre-submission inquiries through Email, Facebook, LinkedIn, Twitter, etc. A wide selection of journals (inclusive of 9 subjects, more than 200 journals)

Providing 24-hour high-quality service

User-friendly online submission system

Fair and swift peer-review system

Efficient typesetting and proofreading procedure

Display of the result of downloads and visits, as well as the number of cited articles Maximum dissemination of your research work

Submit your manuscript at: http://papersubmission.scirp.org/

Or contact health@scirp.org 